\title{
A pilot study of respiratory rate derived from a wearable biosensor compared with capnography in emergency department patients
}

This article was published in the following Dove Press journal: Open Access Emergency Medicine

\author{
Timmy Li' \\ Shreya Divatia ${ }^{2}$ \\ Justine McKittrick ${ }^{3}$ \\ Jeena Moss ${ }^{3}$ \\ Nicole $M$ Hijnen ${ }^{2}$ \\ Lance B Becker' \\ 'Department of Emergency Medicine, \\ Donald and Barbara Zucker School of \\ Medicine at Hofstra/Northwell, \\ Manhasset, NY I I030, USA; \\ 2Department of Connected Sensing, \\ Philips North America Corporation, \\ Cambridge, MA 02I7I, USA; \\ ${ }^{3}$ Department of Emergency Medicine, \\ North Shore University Hospital, \\ Manhasset, NY I 1030, USA
}

Purpose: Respiratory rate is assessed less frequently than other vital signs, and documented respiratory rates are often erroneous. This pilot study compared respiratory rates derived from a wearable biosensor to those derived from capnography.

Methods: Emergency department patients with respiratory complaints were enrolled and had capnography via nasal cannula and a wireless, wearable biosensor from Philips applied for approximately one hour. Respiratory rates were obtained from both of these methods. We determined the difference between median respiratory rates obtained from the biosensor and capnography and the proportion of biosensor-derived respiratory rates that were within three breaths/minute of the capnography-derived respiratory rates for each patient. A Spearman correlation coefficient was calculated to assess the strength of the correlation between mean respiratory rates derived from both methods. Plots of minute-by-minute respiratory rates, per patient, for each monitoring method were shown to two physicians. The physicians identified time periods in which the respiratory rates appeared invalid. The proportion of time with invalid respiratory rates for each patient, for each method, was calculated and averaged.

Results: We analyzed data for 17 patients. Median biosensor-derived respiratory rate was 20 breaths/minute (range: $7-40$ breaths/minute) and median capnography-derived respiratory rate was 25 breaths/minute (range: $0-58$ breaths/minute). Overall, $72.8 \%$ of biosensorderived respiratory rates were within three breaths per minute of the capnography-derived respiratory rates. Overall mean difference was 3.5 breaths/minute $( \pm 5.2$ breaths/minute) Respiratory rates appeared invalid $0.7 \%$ of the time for the biosensor and $5.0 \%$ of the time for capnography.

Conclusion: Our pilot study suggests that the Philips wearable biosensor can continuously obtain respiratory rates that are comparable to capnography-derived respiratory rates among emergency department patients with respiratory complaints.

Keywords: respiratory rate, emergency department, clinical monitoring

\section{Introduction}

Respiratory rates and changes in respiratory rate have been found to be strong predictors of early clinical deterioration, making respiratory rate an important vital sign. ${ }^{1-3}$ However, evidence indicates that respiratory rate is assessed less frequently than other vital signs, and respiratory rates documented in the medical record are often erroneous due to estimation rather than actual measurement. ${ }^{1,4}$

Although capnography is regarded as the gold standard for monitoring respiratory rate in intubated and perioperative patients, ${ }^{8,9}$ there is no gold standard for
Correspondence: Timmy Li

Department of Emergency Medicine, Donald and Barbara Zucker School of

Medicine at Hofstra/Northwell, 300

Community Drive, Manhasset, NY I I030,

USA

Tel +I 5165621513

Email TLi2@northwell.edu 
monitoring respiratory rate in patients who are awake and mobile. Capnography monitoring requires that the patient wear a nasal cannula connected to a capnograph that derives a respiratory rate, ${ }^{2}$ which limits patient mobility. The nasal cannula may be uncomfortable and may be moved or removed by the patient, leading to inaccurate measurements and erroneous alarms.

Manual observation of respiratory rate is standard clinical practice; however, it does not provide continuous monitoring, is prone to error, and is inefficient in busy settings. ${ }^{3,7}$ Therefore, non-intrusive methods of monitoring respiratory rate continuously and accurately are important. The objective of our pilot study was to compare respiratory rates derived from a wearable biosensor from Philips with those derived from capnography.

\section{Methods}

\section{Study design and setting}

We conducted a prospective pilot study of a convenience sample of adult patients with respiratory chief complaints who presented to the emergency department of North Shore University Hospital in Manhasset, New York between July 2017 and December 2017. North Shore University Hospital, part of the Northwell Health system, is a 738-bed tertiary care facility with an emergency department that cares for approximately 90,000 patients per year.

This study was approved by the Northwell Health Institutional Review Board and was conducted in accordance with the Declaration of Helsinki and Good Clinical Practice. Written informed consent was obtained from all subjects prior to completing any research procedures. We included patients who were English-speaking, $\geq 18$ years of age, had an Emergency Severity Index score of 2 or 3, and had a respiratory chief complaint (eg, shortness of breath, difficulty breathing, or dyspnea). We excluded patients who had an implanted defibrillator, pacemaker, or allergy to adhesives.

\section{Philips wearable biosensor}

The Philips wearable biosensor is a wireless, batteryoperated, skin-adhesive device that weighs 12 grams, and measures $115 \mathrm{~mm}$ in length, $36 \mathrm{~mm}$ in width, and $8 \mathrm{~mm}$ in depth. When adhered to a patient's chest, it continuously records and transmits the patient's respiratory rate, heart rate, ambulation, and posture. The biosensor has received Food and Drug Administration 510(k) clearance under
K152139 for in-hospital use. Due to its wireless nature, patient movement is not restricted and patients are able to move away from their bed during the monitoring period.

\section{Study protocol and data collection}

Trained research assistants monitored our hospital's electronic medical record system to track patients in the emergency department. Patients appearing to meet eligibility criteria were approached by a research assistant and the research assistant explained the study to the patient and verified eligibility. Patients were given the opportunity to ask questions as well as review the consent form alone. The research assistant returned after a few minutes and patients interested in participating were enrolled. After written informed consent was obtained, the research assistant applied the wearable biosensor on the patient's chest as well as capnography via nasal cannula. Patients wore the biosensor and capnography for approximately one hour, and minute-by-minute respiratory rates were obtained. All data were stored in a secure, password-protected, electronic database, only accessible by study staff.

\section{Data analysis}

Respiratory rates derived from the biosensor and capnography for all patients, for the entire monitoring period, were qualitatively compared using boxplots. Following the method described by Bergese et al, ${ }^{9}$ capnography-derived respiratory rate was used as the reference and we determined the difference between respiratory rates obtained from the biosensor and capnography. We then determined the proportion of biosensor-derived respiratory rates that were within three breaths/minute of the capnography-derived respiratory rates for each patient, as described by Bergese et $\mathrm{al}^{9}$. A Spearman's correlation coefficient was calculated to assess the strength of the correlation between mean respiratory rates derived from both methods. Plots of minute-byminute respiratory rates, per patient, for each monitoring method were shown to two physicians, dually board certified in emergency medicine and critical care medicine, along with the patient's age, sex, chief complaint, and past medical history. The physicians were blinded to the method in which the respiratory rates were derived, and were asked to identify time periods on the plots in which respiratory rates appeared invalid based on their clinical judgment (eg, respiratory rates of two breaths per minute). The proportion of time with invalid respiratory rates for each patient, for each method, was calculated and averaged. Overall mean difference and proportion of biosensor-derived respiratory rates within three 
breaths/minute of capnography-derived respiratory rates were recalculated with the invalid time periods excluded.

Because this was a pilot study, a sample size calculation was not performed prior to performing this study. A post-hoc power analysis was conducted and revealed that with 17 pairs of subjects, we had $74 \%$ power with a Type I error probability of $5 \%$.

\section{Results}

A total of 23 patients were enrolled, but six patients were excluded from the analysis due to errors in application of the biosensor (eg, did not power on biosensor, did not remove chest hair from patient prior to application of the biosensor, and using an expired biosensor); therefore only 17 patients had analyzable data. Mean age was 61 years, 59\% were male, and median emergency department length of stay was $12.1 \mathrm{hrs}$. A total of 990 minutes of respiratory rates from the biosensor and capnography were available. There was a statistically significant strong correlation between mean respiratory rates derived from both monitoring methods (Spearman's $\rho=0.86275 ; p<0.0001$ ). As shown in Figure 1, median biosensor-derived respiratory rate was 20 breaths/minute (range: $7-40$ breaths/minute) and median capnography-derived respiratory rate was 25 breaths/minute (range: $0-58$ breaths/minute). Overall,
$72.8 \%$ of biosensor-derived respiratory rates were within three breaths per minute of the reference capnographyderived respiratory rates. Mean difference in respiratory rates for each patient is shown in Figure 2. Overall mean difference (mean of mean difference for all patients) was 3.5 breaths/minute ( \pm 5.2 breaths/minute). The majority of capnography-derived respiratory rates were higher than biosensor-derived respiratory rates. Of note, patient \#4 in Figure 2 appears to be an outlier, with substantially higher differences in respiratory rates between the two methods.

Based on the two physicians' blinded review, respiratory rates appeared invalid $0.7 \%$ of the time for the biosensor and $5.0 \%$ of the time for capnography. When these invalid time periods were excluded, overall mean difference between the two methods was 3.2 breaths/minute ( \pm 3.6 breaths/minute) and $76.3 \%$ of biosensor-respiratory rates were within three breaths per minute of the reference capnography-derived respiratory rates.

\section{Discussion}

This pilot study compared respiratory rates derived from a wearable biosensor from Philips with respiratory rates derived from standard capnography via nasal cannula. Results from several studies show that clinical deterioration is often preceded by changes in respiratory rate. ${ }^{1-3,6}$ Therefore, respiratory

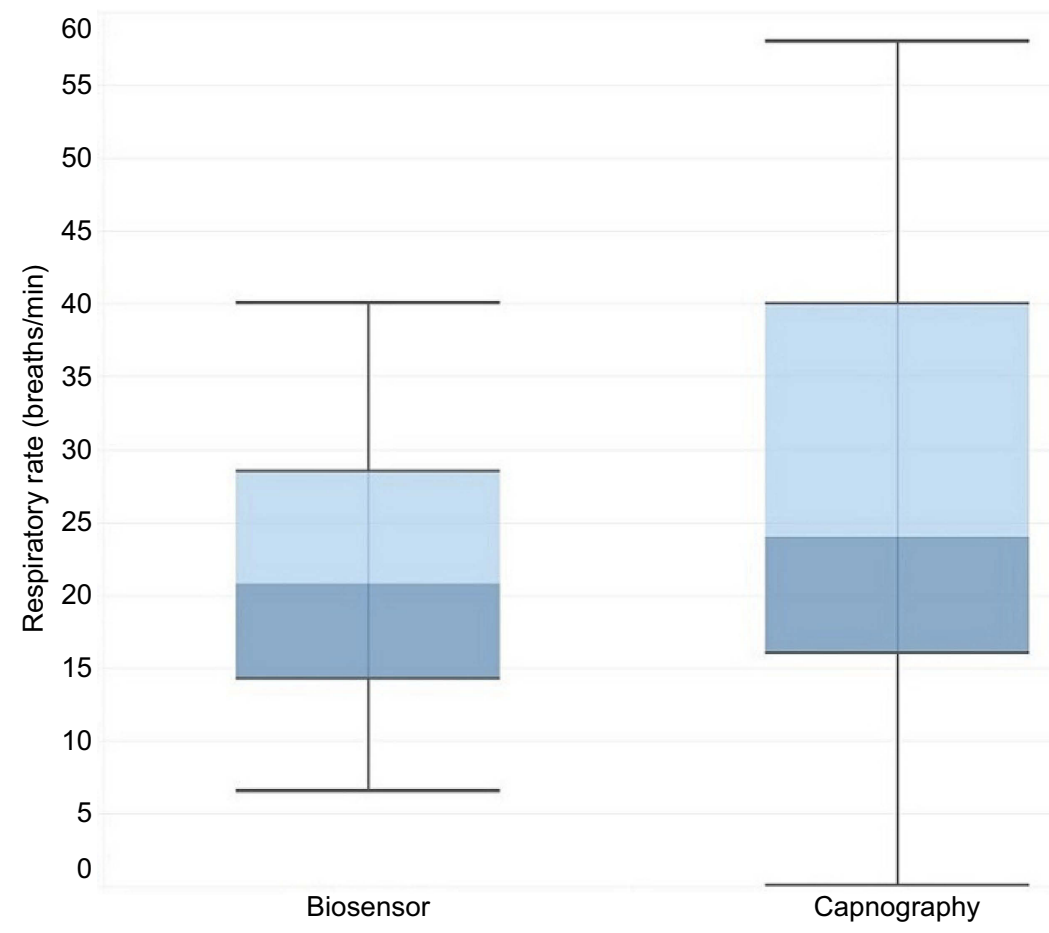

Figure I Distribution of respiratory rates derived from Philips wearable biosensor and capnography ( $\mathrm{n}=\mathbf{1 7})$. Boxplots showing the following values: minimum, quartile I (25th percentile), median (50th percentile), quartile 3 (75th percentile), and maximum. 


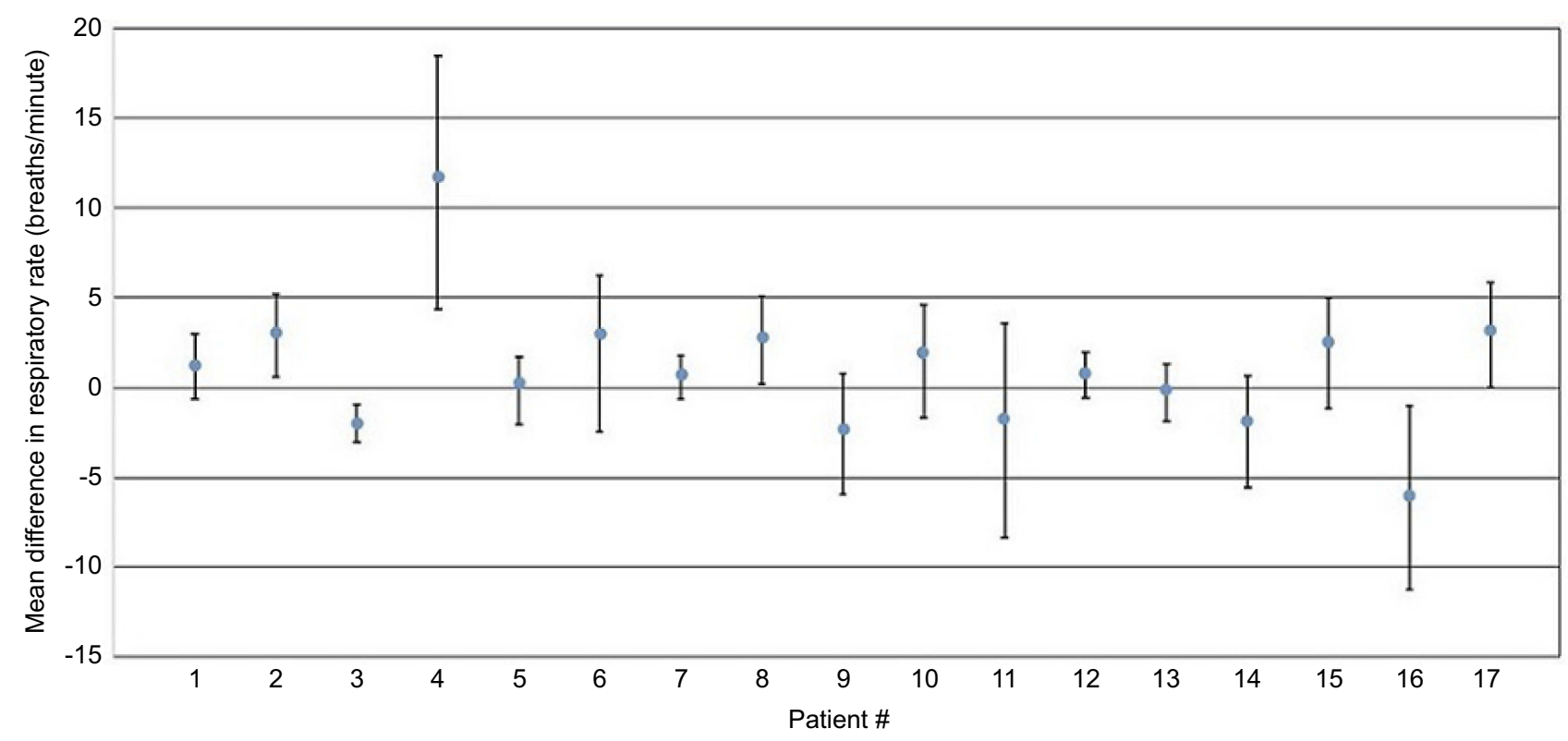

Figure 2 Mean difference in respiratory rate per patient. Difference was calculated by subtracting Philips wearable biosensor-derived respiratory rate from capnographyderived respiratory rate. Mean difference was calculated by averaging the minute-by-minute differences. Error bars represent SDs. Patient \#4 appears to be an outlier.

rate is an important vital sign to monitor. However, respiratory rate is often assessed less frequently compared with other vital signs, and it is known in the medical community that respiratory rates are often estimated, rather than actually measured. ${ }^{1,4-7}$ Further, it is not feasible to obtain frequent respiratory rate measurements in busy clinical settings, such as the emergency department. The gold standard for monitoring respiratory rate in patients who are intubated and patients in perioperative setting is via capnography. ${ }^{8,9}$ However, it may not be practical to apply a nasal cannula on every patient in the emergency department, as it limits movement and may be uncomfortable to wear.

Therefore, non-intrusive methods of continuously monitoring respiratory rate are needed. With accurate respiratory rates, clinical deterioration may be predicted, and interventions may be initiated to prevent deterioration. ${ }^{7}$ Continuous respiratory rate monitoring may be particularly important in the emergency department setting, as patients are often placed in a room and unmonitored for long periods of time. With a reliable and non-intrusive, continuous respiratory rate monitoring method, conditions such as sepsis may be able to be identified early, as many risk stratification and prediction scores include respiratory rate as a parameter. ${ }^{10,11}$ One study found that serial vital sign measurements among patients with suspected infection or sepsis in the emergency department can be used to identify patients at risk for deterioration within 72 hours, and respiratory rate was a significant predictor of deterioration. ${ }^{12}$ Another study found that respiratory rate can be used to predict patient outcomes among acute heart failure patients in the emergency department. ${ }^{13}$ Therefore, reliable and continuous respiratory rate measurements are critically important for patient safety, risk satisfaction, and prediction of clinical outcomes.

The Philips wearable biosensor can be adhered to the chest of patients, is completely wireless, and does not limit movement and thus, may be a viable respiratory rate monitoring method for emergency department patients. Our pilot study found that respiratory rates derived from the biosensor were generally similar to respiratory rates derived from capnography; $72.8 \%$ of the biosensor-derived respiratory rates were within three breaths/minute of the capnography-derived respiratory rates. Respiratory rates from both methods were also strongly correlated (Spearman's $\rho$ : 0.86275). Further, based on physicians' blinded review, there appeared to be more invalid respiratory rates derived from capnography compared with the biosensor $(5.0 \%$ vs $0.7 \%$ ).

A prior study by Subbe et al reported on the performance of a similar wireless respiratory rate monitoring device compared with standard capnography. ${ }^{14}$ This studied enrolled patients admitted to an acute medical unit and found that the device can obtain respiratory rates that are comparable to standard capnography. Our study differs in that we enrolled patients from the emergency department, where patients may 
be unstable and unmonitored for long periods of time. Nevertheless, our results are complementary to those by Subbe et al, and adds to the growing body of literature on wireless respiratory rate monitoring devices.

\section{Limitations}

There are a few limitations of this pilot study to acknowledge. First, our study included a small sample size of only 17 patients. Therefore, a larger study is warranted to verify our findings. Second, we only enrolled patients with respiratory chief complaints. Therefore, whether other physiologic states affect the performance of the wearable biosensor is unknown. Lastly, capnography-derived respiratory rates were used as the reference in this study, as they are currently considered the gold standard. However, capnography often shows noise and errors when obtaining respiratory rates for awake and mobile patients. Hence, although we are able to conclude that biosensor-derived respiratory rates were comparable to respiratory rates derived from capnography, without a true gold standard, we are unable to determine the effect of errors on these findings.

\section{Conclusion}

Our pilot study suggests that the Philips wearable biosensor can continuously obtain respiratory rates that are comparable to capnography-derived respiratory rates among emergency department patients with respiratory complaints. The majority (72.8\%) of biosensor-derived respiratory rates were within three breaths of capnographyderived respiratory rates and biosensor-derived respiratory rates appeared invalid $<1 \%$ of the time. The Philips wearable biosensor appears to be a valid and reliable method of automated respiratory rate monitoring while allowing patients freedom of movement.

\section{Acknowledgment}

This work was funded by Philips North America Corporation, Cambridge, MA, USA.

\section{Disclosure}

SD and NMH are employees of Philips North America Corporation. LBB reports grants from Philips North America Corporation during the conduct of the study and grants from
United Therapeutics, BrainCool, PCORI, Zoll Medical, Nihon Kohden, and NIH, outside the submitted work. The other authors report no conflicts on interest in this work.

\section{References}

1. Mochizuki K, Shintani R, Mori K, et al. Importance of respiratory rate for the prediction of clinical deterioration after emergency department discharge: a single-center, case-control study. Acute Med Surg. 2017;4(2):172-178. doi:10.1002/ams2.252

2. Donnelly N, Hunniford T, Harper R, et al. Demonstrating the accuracy of an in-hospital ambulatory patient monitoring solution in measuring respiratory rate. Conf Proc IEEE Eng Med Biol Soc. 2013;2013:6711-6715. doi:10.1109/EMBC.2013.6611096

3. Addison PS, Watson JN, Mestek ML, Ochs JP, Uribe AA, Bergese SD. Pulse oximetry-derived respiratory rate in general care floor patients. J Clin Monit Comput. 2015;29(1):113-120. doi:10.1007/s10877-014-9575-5

4. Yonge JD, Bohan PK, Watson JJ, Connelly CR, Eastes L, Schreiber MA. The respiratory rate: a neglected triage tool for pre-hospital identification of trauma patients. World J Surg. 2018;42 (5):1321-1326. doi:10.1007/s00268-017-4353-4

5. Parkes R. Rate of respiration: the forgotten vital sign. Emerg Nurse. 2011;19(2):12-17. quiz 18. DOI:10.7748/en2011.05.19.2.12.c8504.

6. Churpek MM, Snyder A, Twu NM, Edelson DP. Accuracy comparisons between manual and automated respiratory rate for detecting clinical deterioration in ward patients. J Hosp Med. 2018. doi:10.12788/jhm.2914

7. Flenady T, Dwyer T, Applegarth J. Accurate respiratory rates count: so should you! Australas Emerg Nurs J. 2017;20(1):45-47. doi:10.1016/j.aenj.2016.12.003

8. Touw HRW, Verheul MH, Tuinman PR, et al. Photoplethysmography respiratory rate monitoring in patients receiving procedural sedation and analgesia for upper gastrointestinal endoscopy. J Clin Monit Comput. 2017;31(4):747-754. doi:10.1007/s10877-016-9890-0

9. Bergese SD, Mestek ML, Kelley SD, et al. Multicenter study validating accuracy of a continuous respiratory rate measurement derived from pulse oximetry: a comparison with capnography. Anesth Analg. 2017;124(4):1153-1159. doi:10.1213/ANE.0000000000001852

10. Singer M, Deutschman CS, Seymour CW, et al. The third international consensus definitions for sepsis and septic shock (Sepsis-3). JAMA. 2016;315(8):801-810. doi:10.1001/jama.2016.0287

11. Gardner-Thorpe J, Love N, Wrightson J, Walsh S, Keeling N. The value of modified early warning score (MEWS) in surgical in-patients: a prospective observational study. Ann $R$ Coll Surg Engl. 2006;88(6):571-575. doi:10.1308/003588406X130615

12. Quinten VM, van Meurs M, Olgers TJ, Vonk JM, Ligtenberg JJM, Ter Maaten JC. Repeated vital sign measurements in the emergency department predict patient deterioration within 72 hrs: a prospective observational study. Scand J Trauma Resusc Emerg Med. 2018;26 (1):57. doi:10.1186/s13049-018-0525-y

13. Siniorakis E, Arvanitakis S, Tsitsimpikou C, et al. Acute heart failure in the emergency department: respiratory rate as a risk predictor. In Vivo. 2018;32(4):921-925. doi:10.21873/ invivo. 11330

14. Subbe CP, Kinsella S. Continuous monitoring of respiratory rate in emergency admissions: evaluation of the respirasense sensor in acute care compared to the industry standard and gold standard. Sensors (Basel). 2018;18(8). doi:10.3390/s18082700 


\section{Publish your work in this journal}

The Open Access Emergency Medicine is an international, peerreviewed, open access journal publishing original research, reports, editorials, reviews and commentaries on all aspects of emergency medicine. The manuscript management system is completely online and includes a very quick and fair peer-review system, which is all easy to use. Visit http://www.dovepress.com/testimonials.php to read real quotes from published authors.

Submit your manuscript here: https://www.dovepress.com/open-access-emergency-medicine-journal 